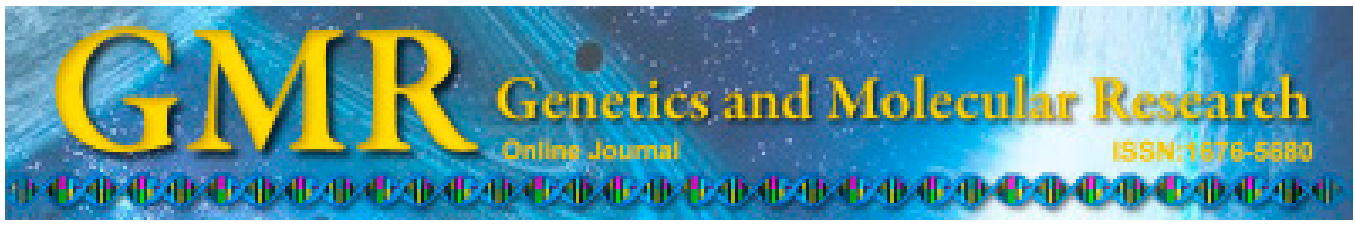

\title{
Cyclin D1 G870A polymorphism is associated with an increased risk of multiple myeloma
}

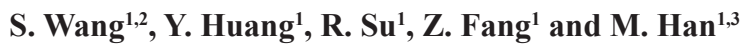 \\ ${ }^{1}$ Department of Hematology, The First Affiliated Hospital of Xiamen University, \\ Xiamen, China \\ ${ }^{2}$ Department of Hematology, \\ First Clinical Medical College of Fujian Medical University, Fuzhou, China \\ ${ }^{3}$ Center of Hematopoietic Stem Cell Transplantation, \\ Institute of Hematology and Hospital of Blood Diseases, \\ Chinese Academy of Medical Sciences and Peking Union Medical College, \\ Tianjin, China
}

Corresponding author: M. Han

Email: mzhan@medmail.com.cn

Genet. Mol. Res. 14 (2): 5856-5861 (2015)

Received May 5, 2014

Accepted November 10, 2014

Published June 1, 2015

DOI http://dx.doi.org/10.4238/2015.June.1.2

ABSTRACT. Cyclin D1 is an important cell cycle regulator implicated
in the pathogenesis of many cancer types. In particular, translocation and
overexpression of cyclin D1 are common events in multiple myeloma
(MM), suggesting that it may drive the initiation and progression of this
malignancy. However, the association between genetic polymorphisms
of cyclin D1 and the risk for developing MM remains poorly
characterized. We performed a case-control study with 67 patients
with MM and 66 healthy controls in a Chinese population. The cancer-
associated G870A single nucleotide polymorphism (SNP) of CCND1,
the gene encoding cyclin D1, was determined by polymerase chain
reaction and restriction fragment length polymorphism. We found that
there was a strong association between the homozygous variant allele 
(GG) and MM susceptibility, with an odds ratio (OR) of 4.679 [95\% confidence interval (CI): 1.081-5.647]. When further stratified analysis was performed, the increased risk was only evident in individuals over 60 years old $(\mathrm{OR}=3.297 ; 95 \% \mathrm{CI}: 1.058-10.276)$. Our results suggest that the CCND1 G870A SNP may be critically involved in MM development.

Key words: Single nucleotide polymorphism; Cyclin D1; Multiple myeloma

\section{INTRODUCTION}

Multiple myeloma (MM) is a neoplasm originating from monoclonal plasma cells. It is the second most common hematological malignancy and represents $1 \%$ of all cancers (Raab et al., 2009). Despite recent advances in the treatment of MM, the prognosis is poor and the genetic and molecular mechanisms underlying MM development remain unclear.

Deregulation of the cell cycle plays an important role in malignant transformation (Sherr, 1996). During mitosis, the transition of the cell cycle from G1 into S phase is controlled by the cyclin-dependent kinases (CDKs) CDK4 and CDK6, in protein complexes with cyclin D1 (Hunter and Pines, 1994). Cyclin D1 catalyzes the phosphorylation of the tumor suppressor protein retinoblastoma (RB), which in turn releases the transcriptional factor E2F and activates the transcription of downstream genes necessary for cell cycle progression. Therefore, inhibition of cyclin D1 causes cell cycle arrest, whereas overexpression of the protein accelerates the G1 phase transition. Abnormal expression of cyclin D1 due to chromosome amplification, translocation, and inversion has been reported in various human cancers (Bartkova et al., 1997). In particular, 15-20\% of MM samples harbor the $t(11 ; 14)$ chromosomal translocation, which leads to aberrant transcriptional activation of CCND1, the gene encoding cyclin D1 (Sawyer, 2011). Overexpression of cyclin D1 was also found in 25-50\% of MM samples (Specht et al., 2004). These results suggest that deregulation of cyclin D1 and the cell cycle may be a critical "driver" event in MM development.

In addition to genetic mutations and chromosomal abnormality, a number of single nucleotide polymorphisms (SNPs) in the CCND1 gene such as G870A at the exon 4 splice site were associated with an increased risk and poor prognosis in several types of tumors (Cui et al., 2012; Yang et al., 2012; Zhou et al., 2013; Wang et al., 2014). However, the link between CCND1 SNPs and MM susceptibility is unclear. In this case-control study, we quantified the CCND1 G870A allele frequencies in patients with $\mathrm{MM}$ and controls and determined the association between the G870A SNP and the risk for developing MM in a Chinese population.

\section{MATERIAL AND METHODS}

\section{Study subjects}

Cases included a total of 67 patients with histologically confirmed MM who had been admitted to the First Affiliated Hospital of Xiamen University from January 2007 to December 2010 . Healthy controls $(N=66)$ who had no history of cancer were recruited from the individuals attending the clinic for annual health check-ups during August 2010 and December 2010. 
Controls were matched to the patients with MM by age, gender, and ethnicity. All participants were asked to complete a questionnaire to collect epidemiological data, including demographical information. Blood samples were obtained in heparinized tubes for genetic analyses. For both patients and controls, informed consent was obtained from each participant and the study protocol was approved by the institutional review board.

\section{Genotyping by restriction fragment length polymorphism}

Genomic DNA was extracted from peripheral blood lymphocytes using a commercially available kit (Promega, Madison, WI, USA) according to manufacturer instructions. DNA samples were stored at $-80^{\circ} \mathrm{C}$ until genotyped. CCND1 G870A genotyping was performed using the polymerase chain reaction (PCR). Primers for amplification of CCND1 were as follows: 5'-GCA GTG CAA GGC CTG AAC CT - 3' (sense) and 5'-GGG ACA TCA CCC TCA CTT AC - 3' (antisense). PCR was performed in $30 \mu \mathrm{L}$ volumes containing $1 \mathrm{U}$ Taq polymerase, $5 \mathrm{ng}$ genomic DNA, $10 \mathrm{nM}$ of each primer, and $2.5 \mu \mathrm{M}$ dNTPs. Thermocycling was carried out at $94^{\circ} \mathrm{C}$ for $5 \mathrm{~min}$ followed by 35 cycles at $94^{\circ} \mathrm{C}$ for $60 \mathrm{~s}, 60^{\circ} \mathrm{C}$ for $60 \mathrm{~s}$, and $72^{\circ} \mathrm{C}$ for $1 \mathrm{~min}$, with a final incubation at $72^{\circ} \mathrm{C}$ for $7 \mathrm{~min}$. After confirmation of the 167-bp PCR fragment by $1.5 \%$ agarose gel electrophoresis, each PCR product was digested overnight with $5 \mathrm{U} \mathrm{ScrFI}$ at $37^{\circ} \mathrm{C}$ (New England Biolabs Inc., Beverly, MA, USA) and separated by $3.0 \%$ agarose gel electrophoresis. The 167-bp PCR fragment was digested into 146- and 22-bp fragments when the $S c r$ FI site was present (Simpson et al., 2001). The genotype was designated as $\mathrm{G}$ or A when the $S c r$ FI restriction site was present or absent, respectively.

\section{Statistical analysis}

All statistical analyses were performed with the SPSS 10 statistical software package (SPSS, Chicago, IL, USA). Pearson's $\chi^{2}$-test was used to compare the distribution of the $C C N D 1$ genotypes between the cases and the controls. The Student $t$-test was used to test for differences in the distribution of ages between the two groups. Hardy-Weinberg equilibrium was tested using the goodness-of-fit $\chi^{2}$-test to compare the observed allele frequencies with the expected frequencies in control subjects. Unconditional multivariate logistic regression models were used to calculate the odds ratio [OR; 95\% confidence interval (CI)] associated with the CCND1 genotypes while controlling for the possible confounding factor (age). All statistical tests were two-sided with a significance level of $\mathrm{P}>0.05$.

\section{RESULTS}

The analysis included 67 patients with MM and 66 controls. The demographic variables are shown in Table 1. There were no statistically significant differences between patients and controls in terms of gender or age.

Genotype frequencies, as well as ORs for MM risk for all SNPs are shown in Table 2. The prevalence of the homozygous GG genotype was $26 \%(17 / 66)$ in the controls and $42 \%$ $(28 / 67)$ in the patients. The heterozygous GA genotype was identified in $29 \%(19 / 66)$ of the controls and in $28 \%(19 / 67)$ of the patients. The homozygous AA genotype was found in $45 \%$ $(30 / 66)$ of the controls and $30 \%(20 / 67)$ of the patients. The genotype frequencies of both control and patient groups did not deviate from Hardy-Weinberg equilibrium. The homozygous 
GG genotype was significantly associated with increased risk for $\mathrm{MM}$ development $(\mathrm{OR}=$ $4.679,95 \% \mathrm{CI}=1.081-5.647, \mathrm{P}=0.031)$.

Stratified analyses were performed to examine the effect of age on the association between CCND1 SNPs and MM susceptibility. We found that the increased risk conferred by the GG genotype was evident in individuals older than 60 years $(\mathrm{OR}=3.297 ; 95 \% \mathrm{CI}=1.058$ $10.267, \mathrm{P}=0.036)$ but not younger than 60 years $(\mathrm{OR}=0.555 ; 95 \% \mathrm{CI}=0.544-3.862$; Table 2$)$.

Table 1. Demographic parameters of patient and control groups.
\begin{tabular}{lcc}
\hline Parameters & Patients & Controls \\
& $(\mathrm{N}=67)$ & $(\mathrm{N}=66)$ \\
\hline Gender (male/female) & $44 / 23$ & $36 / 30$ \\
Age (years) (means \pm SD) & $55.955 \pm 10.574$ & $53.682 \pm 9.858$ \\
Ethnicity (\% of Han) & 100 & 100 \\
\hline
\end{tabular}

Table 2. CCND1genotype and allele frequencies of patient and control groups.

\begin{tabular}{|c|c|c|c|c|c|c|c|}
\hline & \multicolumn{2}{|c|}{ Patients $(\mathrm{N}=67)$} & \multicolumn{2}{|c|}{ Controls $(\mathrm{N}=66)$} & \multirow[b]{2}{*}{ OR } & \multirow[b]{2}{*}{$95 \% \mathrm{CI}$} & \multirow[b]{2}{*}{$\mathrm{P}$} \\
\hline & $\mathrm{N}$ & $\%$ & $\mathrm{~N}$ & $\%$ & & & \\
\hline \multicolumn{8}{|l|}{ Allele } \\
\hline A & 59 & 44 & 79 & 60 & 1.00 & reference & \\
\hline G & 75 & 56 & 53 & 40 & 1.895 & $1.164-3.084$ & $0.01 *$ \\
\hline \multicolumn{8}{|l|}{ Genotype } \\
\hline $\mathrm{AA}$ & 20 & 30 & 30 & 45 & 1.00 & reference & \\
\hline $\mathrm{AG}$ & 19 & 28 & 19 & 29 & 0.875 & $0.64-3.514$ & 0.350 \\
\hline GG & 28 & 42 & 17 & 26 & 4.679 & $1.081-5.647$ & 0.031 \\
\hline \multicolumn{8}{|l|}{ Age } \\
\hline \multicolumn{8}{|l|}{$<60$ years } \\
\hline $\mathrm{AA} / \mathrm{AG}$ & 26 & 39 & 29 & 44 & 1.00 & reference & \\
\hline $\mathrm{GG}$ & 13 & 19 & 10 & 15 & 0.555 & $0.544-3.862$ & 0.456 \\
\hline \multicolumn{8}{|l|}{$\geq 60$ years } \\
\hline $\mathrm{AA} / \mathrm{AG}$ & 13 & 19 & 20 & 30 & 1.00 & reference & \\
\hline GG & 15 & 23 & 7 & 11 & 3.297 & $1.058-10.276$ & $0.036^{*}$ \\
\hline
\end{tabular}

$\mathrm{OR}=$ odds ratio; $\mathrm{CI}=$ confidence interval. *Statistically significant.

\section{DISCUSSION}

MM is a neoplasm originating from terminally differentiated plasma cells. Normal plasma cells are arrested in the G1 phase of the cell cycle. In contrast, MM cells acquire the abilities to proliferate and self-renew, suggesting that their cell cycle control is impaired through genetic and/or epigenetic mechanisms. Indeed, overexpression of cyclin D1, a critical regulator of G1/S transition, is commonly found in MM (Specht et al., 2004). Furthermore, one of the most common translocations in $\mathrm{MM}, \mathrm{t}(11 ; 14)(\mathrm{q} 13 ; \mathrm{q} 32)$, involves abnormal fusion of the immunoglobulin heavy chain locus to cyclin D1 (Sawyer, 2011). Our results support the existing literature documenting a pivotal role of cyclin D1 in MM development by demonstrating a strong association between CCND1 SNPs and MM susceptibility in a Chinese population. Our findings are consistent with the recent report proposing that the CCND1 G870A polymorphism is a risk factor for MM in two European patient cohorts (Weinhold et al., 2013). Furthermore, in the current study we performed stratified analysis and showed that age is an important determinant of the association between CCND1 SNPs and the risk for MM develop- 
ment. Hence, our study suggests that genotyping of cyclin D1 combined with demographic parameters (age) may provide useful clinical information for evaluating an individual's risk for MM development.

In addition to MM, CCND1 SNPs have been linked to various human malignancies. For example, several meta-analyses studies have reported associations of the CCND1 G870A genetic polymorphism with increased susceptibilities to colorectal, lung, breast, and bladder cancers (Cui et al., 2012; Yang et al., 2012; Zhou et al., 2013; Wang et al., 2014). Notably, while the majority of previous studies observed that the AA genotype contributed to increased cancer risk, for MM, the GG genotype seemed to confer increased risk as reported here and by others (Weinhold et al., 2013). The underlying molecular mechanism(s) for our populationbased findings remain unclear and require further laboratory investigation. The G/A 870 polymorphism is located at the boundary of intron 4 and exon 5 of CCND1. It is believed that the G allele results in the "canonical" cyclin D1a transcript, whereas the A allele hinders the splicing process and produces the alternatively spliced cyclin D1b (Solomon et al., 2003). Additional study is needed to determine whether MM samples from patients with the GG genotype lack the expression of cyclin D1b mRNA and protein. The differential roles of cyclin D1 variants in tumorigenesis are increasingly appreciated (Knudsen et al., 2006). It was shown that cyclin D1b was preferentially retained in the nucleus and exhibited higher transformation capacity (Lu et al., 2003). On the other hand, cyclin D1b might also act as a potential tumor suppressor by modulating cellular response to DNA damage ( $\mathrm{Li}$ et al., 2010). Impaired DNA damage response would facilitate the accumulation of genetic aberrations overtime, which is consistent with our observation that the association of the CCND1 G870A polymorphism with MM risk is positively correlated with age.

In conclusion, our study demonstrated a novel age-dependent association between the cyclin D1 G870A polymorphism and increased risk for developing MM. Independent casecontrol studies with larger sample size are required to validate the findings presented here. Additional studies on gene-gene and gene-environment interactions are also warranted. Our results suggest that CCND1 G870A SNP variation may be a critical mechanism by which normal plasma cells escape cell cycle surveillance and undergo malignant transformation, leading to the development of MM.

\section{ACKNOWLEDGMENTS}

Research supported by grants to M. Han from the Tianjin Research Program of the Application Foundation and Advanced Technology (\#10JCYBJC11200) and the National Natural Science Foundation for young scholars of China (\#81200364).

\section{REFERENCES}

Bartkova J, Lukas J and Bartek J (1997). Aberrations of the G1- and G1/S-regulating genes in human cancer. Prog. Cell Cycle Res. 3: 211-220.

Cui J, Shen L and Wang Y (2012). Specific CCND1 G870A alleles associated with breast cancer susceptibility: a metaanalysis of 5528 cases and 5353 controls. Asian Pac. J. Cancer Prev. 13: 5023-5025.

Hunter T and Pines J (1994). Cyclins and cancer II: cyclin D and CDK inhibitors come age. Cell 79: 573-582.

Knudsen KE, Diehl JA, Haiman CA and Knudsen ES (2006). Cyclin D1: polymorphism, aberrant splicing and cancer risk. Oncogene 25: 1620-1628.

Li Z, Jiao X, Wang C, Shirley LA, et al. (2010). Alternative cyclin D1 splice forms differentially regulate the DNA damage 
response. Cancer Res. 70: 8802-8811.

Lu F, Gladden AB and Diehl JA (2003). An alternatively spliced cyclin D1 isoform, cyclin D1b, is a nuclear oncogene. Cancer Res. 63: 7056-7061.

Raab MS, Podar K, Breitkreutz I, Richardson PG, et al. (2009). Multiple myeloma. Lancet 374: 324-339.

Sawyer JR (2011). The prognostic significance of cytogenetics and molecular profiling in multiple myeloma. Cancer Genet. 204: 3-12.

Sherr CJ (1996). Cancer cell cycles. Science 274: 1672-1677.

Simpson DJ, Fryer AA, Grossman AB, Wass JA, et al. (2001). Cyclin D1 (CCND1) genotype is associated with tumour grade in sporadic pituitaryadenomas. Carcinogenesis 22: 1801-1807.

Solomon DA, Wang Y, Fox SR, Lambeck TC, et al. (2003). Cyclin D1 splice variants. Differential effects on localization, RB phosphorylation, and cellular transformation. J. Biol. Chem. 278: 30339-30347.

Specht K, Haralambieva E, Bink K, Kremer M, et al. (2004). Different mechanisms of cyclin D1 overexpression in multiple myeloma revealed by fluorescence in situ hybridization and quantitative analysis of mRNA levels. Blood 104: 1120-1126.

Wang Y, Li Z, Liu N and Zhang G (2014). Association between CCND1 and XPC polymorphisms and bladder cancer risk: a meta-analysis based on 15 case-control studies. Tumour Biol. 35: 3155-3165.

Weinhold N, Johnson DC, Chubb D, Chen B, et al. (2013). The CCND1 c.870G $>$ A polymorphism is a risk factor for $\mathrm{t}(11 ; 14)(\mathrm{q} 13 ; \mathrm{q} 32)$ multiple myeloma. Nat. Genet. 45: 522-525.

Yang Y, Wang F, Shi C, Zou Y, et al. (2012). Cyclin D1 G870A polymorphism contributes to colorectal cancer susceptibility: evidence from a systematic review of 22 case-control studies. PLoS One 7: e36813.

Zhou C, An H, Hu M, Liu Q, et al. (2013). The cyclin D1 (CCND1) G870A polymorphism and lung cancer susceptibility: a meta-analysis. Tumour Biol. 34: 3831-3837. 